\title{
Multiplicity Distributions and Charged-neutral Fluctuations
}

\author{
Tapan K. Nayak ${ }^{c}$ 円
}

(for the WA98 Collaboration)

M.M. Aggarwal ${ }^{a}$, A. Agnihotri ${ }^{b}$, Z. Ahammed $^{c}$, A.L.S. Angelis ${ }^{d}$, V. Antonenko ${ }^{e}$, V. Arefiev ${ }^{f}$, V. Astakhov ${ }^{f}$, V. Avdeitchikov ${ }^{f}$, T.C. Awes ${ }^{g}$, P.V.K.S. Baba ${ }^{h}$, S.K. Badyal ${ }^{h}$, A. Baldine ${ }^{f}$, L. Barabach ${ }^{f}$, C. Barlag ${ }^{i}$, S. Bathe ${ }^{i}$, B. Batiounia ${ }^{f}$, T. Bernier ${ }^{j}$, K.B. Bhalla ${ }^{b}$, V.S. Bhatia ${ }^{a}$, C. Blume ${ }^{i}$, R. Bock ${ }^{k}$, E.-M. Bohne ${ }^{i}$, D. Bucher ${ }^{i}$, A. Buijs ${ }^{l}$, E.-J. Buis ${ }^{l}$, H. Büsching ${ }^{i}$, L. Carlen $^{m}$, V. Chalyshev ${ }^{f}$, S. Chattopadhyay ${ }^{c}$, K.E. Chenawi ${ }^{m}$, R. Cherbatchev ${ }^{e}$, T. Chujo $^{n}$, A. Claussen ${ }^{i}$, A.C. Das ${ }^{c}$, M.P. Decowski ${ }^{l}$, V. Djordjadze ${ }^{f}$, P. Donni ${ }^{d}$, I. Doubovik ${ }^{e}$, A.K. Dubey ${ }^{s}$, M.R. Dutta

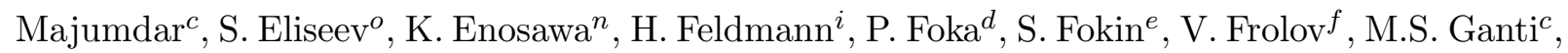
S. Garpman ${ }^{m}$, O. Gavrishchuk ${ }^{f}$, F.J.M. Geurts ${ }^{l}$, T.K. Ghosh ${ }^{p}$, R. Glasow ${ }^{i}$, S.K. Gupta ${ }^{b}$, B. Guskov ${ }^{f}$, H.A. Gustafsson ${ }^{m}$, H.H. Gutbrod ${ }^{j}$, R. Higuchi ${ }^{n}$, I. Hrivnacova ${ }^{o}$, M. Ippolitov ${ }^{e}$, H. Kalechofsky $^{d}$, R. Kamermans ${ }^{l}$, K.-H. Kampert ${ }^{i}$, K. Karadjev ${ }^{e}$, K. Karpio ${ }^{q}$, S. Kato ${ }^{n}$, S. Kees ${ }^{i}$, H. Kim$^{g}$, B.W. Kolb ${ }^{k}$, I. Kosarev ${ }^{f}$, I. Koutcheryaev ${ }^{e}$, A. Kugler ${ }^{o}$, P. Kulinich ${ }^{r}$, V. Kumar ${ }^{b}$, M. Kurata ${ }^{n}$, K. Kurita ${ }^{n}$, N. Kuzmin ${ }^{f}$, I. Langbein ${ }^{k}$, A. Lebedev ${ }^{e}$, Y.Y. Lee ${ }^{k}$, H. Löhner ${ }^{p}$, D.P. Mahapatra ${ }^{s}$,

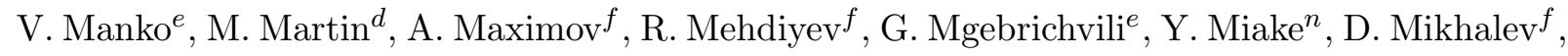
G.C. Mishra $^{s}$, Y. Miyamoto ${ }^{n}$, B. Mohanty ${ }^{s}$, D. Morrison ${ }^{t}$, D.S. Mukhopadhyay ${ }^{c}$, V. Myalkovski $^{f}$, H. Naef ${ }^{d}$, B.K. Nandi ${ }^{s}$, S.K. Nayak ${ }^{j}$, T.K. Nayak ${ }^{c}$, S. Neumaier ${ }^{k}$, A. Nianine ${ }^{e}$, V. Nikitine ${ }^{f}$, S. Nikolaev ${ }^{e}$, S. Nishimura ${ }^{n}$, P. Nomokov $^{f}$, J. Nystrand ${ }^{m}$, F.E. Obenshain ${ }^{t}$, A. Oskarsson ${ }^{m}$, I. Otterlund ${ }^{m}$, M. Pachr ${ }^{o}$, A. Parfenov ${ }^{f}$, S. Pavliouk ${ }^{f}$, T. Peitzmann ${ }^{i}$, V. Petracek ${ }^{o}$, F. Plasil ${ }^{g}$, M.L. Purschke ${ }^{k}$, B. Raeven ${ }^{l}$, J. Rak $^{o}$, R. Raniwala ${ }^{b}$, S. Raniwala ${ }^{b}$, V.S. Ramamurthy ${ }^{s}$, N.K. Rao ${ }^{h}$, F. Retiere ${ }^{j}$, K. Reygers ${ }^{i}$, G. Roland $^{r}$, L. Rosselet $^{d}$, I. Roufanov ${ }^{f}$, J.M. Rubio ${ }^{d}$, S.S. Sambyal ${ }^{h}$, R. Santo ${ }^{i}$, S. Sato ${ }^{n}$, H. Schlagheck ${ }^{i}$, H.-R. Schmidt ${ }^{k}$, G. Shabratova ${ }^{f}$, I. Sibiriak ${ }^{e}$, T. Siemiarczuk $^{q}$, B.C. Sinha ${ }^{c}$, N. Slavine ${ }^{f}$, K. Söderström ${ }^{m}$, N. Solomey ${ }^{d}$, G. Sood $^{a}$, S.P. Sørensen ${ }^{t}$, P. Stankus ${ }^{g}$, G. Stefanek ${ }^{q}$, P. Steinberg ${ }^{r}$, E. Stenlund ${ }^{m}$, D. Stüken ${ }^{i}$, M. Sumbera ${ }^{o}$, T. Svensson ${ }^{m}$, M.D. Trivedi ${ }^{c}$, A. Tsvetkov ${ }^{e}$, C. Twenhöfel ${ }^{l}$, L. Tykarski ${ }^{q}$, J. Urbahn $^{k}$, N.v. Eijndhoven ${ }^{l}$, W.H.v. Heeringen ${ }^{l}$, G.J.v. Nieuwenhuizen ${ }^{r}$, A. Vinogradov ${ }^{e}$, Y.P. Viyogi ${ }^{c}$, A. Vodopianov ${ }^{f}$, S. Vörös ${ }^{d}$, M.A. $\operatorname{Vos}^{l}$, B. Wyslouch ${ }^{r}$, K. Yagi ${ }^{n}$, Y. Yokota ${ }^{n}$, and G.R. Young ${ }^{g}$

${ }^{a}$ Univ. of Panjab (India) ${ }^{b}$ Univ. of Rajasthan (India) ${ }^{c} V E C C$, Calcutta (India) ${ }^{d}$ Univ. of Geneva (Switzerland) ${ }^{e}$ Kurchatov (Russia) ${ }^{f} J I N R$, Dubna (Russia) ${ }^{g}$ ORNL, Oak Ridge (USA) ${ }^{h}$ Univ. of Jammu (India) ${ }^{i}$ Univ. of Münster (Germany) ${ }^{j}$ SUBATECH, Nantes (France) ${ }^{k}$ GSI, Darmstadt (Germany) ${ }^{l}$ NIKHEF, Utrecht (The Netherlands) ${ }^{m}$ Univ. of Lund (Sweden) ${ }^{n}$ Univ. of Tsukuba (Japan) ${ }^{o}$ NPI, Rez (Czech Rep.) ${ }^{p}$ KVI, Groningen (The Netherlands) ${ }^{q} I N S$, Warsaw (Poland) ${ }^{r}$ MIT, Cambridge (USA) ${ }^{s} I O P$, Bhubaneswar (India) ${ }^{t}$ Univ. of Tennessee (USA)

1email : nayak@veccal.ernet.in 
Abstract: Results from the multiplicity distributions of inclusive photons and charged particles, scaling of particle multiplicities, event-by-event multiplicity fluctuations, and chargedneutral fluctuations in $158 \cdot A \mathrm{GeV} \mathrm{Pb}+\mathrm{Pb}$ collisions are presented and discussed. $\mathrm{A}$ scaling of charged particle multiplicity as $N_{\text {part }}^{1.07 \pm 0.05}$ and photons as $N_{\text {part }}^{1.12 \pm 0.03}$ have been observed, indicating violation of naive wounded nucleon model. The analysis of localized charged-neutral fluctuation indicates a model-independent demonstration of non-statistical fluctuations in both charged particles and photons in limited azimuthal regions. However, no correlated chargedneutral fluctuations are observed.

\section{Introduction}

The year 2000 has been quite interesting and remarkable for heavy ion physicists searching for a new form of matter, the quark-gluon plasma (QGP). In the beginning of the year, CERN has announced that there is definite evidence of possible QGP signals from the experiments performed at the SPS [四]. This declaration was made after scrutinizing results from all the dedicated experiments which have been taking data since 1994 using the $\mathrm{Pb}$ beams at $158 \mathrm{GeV} /$ nucleon (a total energy of $33 \mathrm{TeV}$ ). In the middle of the year, this field entered a new era with the commissioning of the Relativistic Heavy Ion Collider (RHIC) at BNL which is a captive machine for QGP search. Soon after, new results from the collisions at RHIC have started to appear [2]. At this time, it is most appropriate to review one of the basic observables of the nuclear collisions, that is the particle multiplicity distributions. One of the distinct advantages of the heavy ion collisions at such high energies is the production of large number of particles in every event. This allows for a detailed study of event-by-event fluctuations in particle multiplicities and ratios of identified particles. This is important for understanding the evolution of nuclear system at high energy collisions. Formation of disoriented chiral condesates (DCC), which is a direct consequence of chiral symmetry restoration, would also give rise to event-by-event correlated fluctuations in charged particles to neutrals. In this manuscript we discuss these topics in view of the data from the WA98 experiment at the CERN-SPS.

For a thermalized system undergoing a phase transition, the variation of the temperature with entropy density is interesting as the temperature is expected to increase below the transition, remain constant during the transition, and then increase again [3, [1]. This can be studied by measuring the mean transverse momentum, $\left\langle p_{T}\right\rangle$, and particle multiplicities for varying centrality, for a number of colliding systems at different energies. Since multiplicity of produced particles is an important quantity to characterize the evolving system, its fluctuation from event to event may provide a distinct signal of the phase transition from hadronic gas to QGP phase. Recently, several new methods using eventby-event fluctuations of $\left\langle p_{T}\right\rangle$ and particle multiplicities have been proposed to probe into the mechanism of phase transition [5 8]. Another interesting phenomenon is the formation of domains of DCC [9 12 which gives rise to isospin fluctuation, in which the neutral pion fraction can deviate significantly from $1 / 3$, the value for uncorrelated emission of

pions. Several methods have been proposed to search for signals of DCC [13 [17]. The most direct signal comes from the event-by-event fluctuations in the number of charged particles to photons in localized $(\eta-\phi)$ phase space.

The observed fluctuations will have contributions from statistical fluctuations and those which have dynamical origin. The contribution from dynamical origin comprise of (a) 

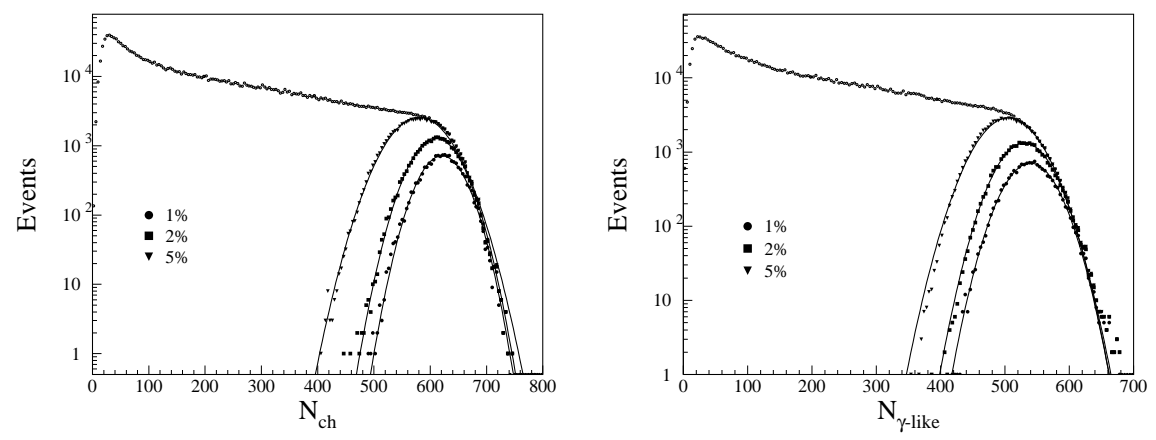

Figure 1. Minimum bias multiplicity distributions of charged particle and photon-like clusters for $\mathrm{Pb}+\mathrm{Pb}$ reactions at $158 \cdot A \mathrm{GeV}$. The other three curves show multiplicity distributions for three different centralities, $0-1 \%, 0-2 \%$ and $0-5 \%$.

fluctuations which do not change event-by-event, e.g., those from Bose-Einstein (BE) correlations, resonance decays and (b) the fluctuations which have new physics origin and may vary from event-to-event. The major interest for us would be to probe into the fluctuations which have new physics origin, such as those arising near the tricritical point of QCD phase diagram and the formation of DCC. To extract the dynamical part from the observed fluctuations, one has to understand the contributions from statistical and other known sources. It is possible to probe at the non-statistical fluctuations from experimental data in a model independent way by comparing these with mixed events generated from the data. Once properly understood, mixed events provide the best means to infer about the presence of non-statistical fluctuations.

This manuscript is organized as follows. In the next section, we discuss multiplicities of charged particles and photons and their pseudorapidity distributions. In section 3 we discuss the scaling of particle multiplicities with centrality of the reaction expressed in terms of the number of participants. In section 4 we present multiplicity fluctuations and discuss the importance of making proper centrality selection. In section 5 we discuss chargedneutral fluctuations in the full phase space of the multiplicity detectors. In section 6 we present the results of an analysis to search for localized fluctuations in smaller $\eta-\phi$ bins by comparing with several kinds of mixed events. We give a summary in section 7 .

\section{Multiplicity Distributions}

The WA98 experimental setup consists of large acceptance hadron and photon spectrometers, detectors for charged particle and photon multiplicity measurements and calorimeters for measuring transverse and forward energies. The charged particle hits were counted using a circular silicon pad multiplicity detector (SPMD) located $32.8 \mathrm{~cm}$ from the target covering $2.35<\eta<3.75$. The efficiency of detecting charged particles has been determined in a test beam to be $99 \%$. The detector is transparent to high energy photons. The photon multiplicity was measured using a preshower Photon Multiplicity Detector (PMD) 19 placed 21.5 meters downstream from the target and covering the pseudorapidity range $2.9<\eta<4.2$. The cluster of hit pads, having total ADC content above a 

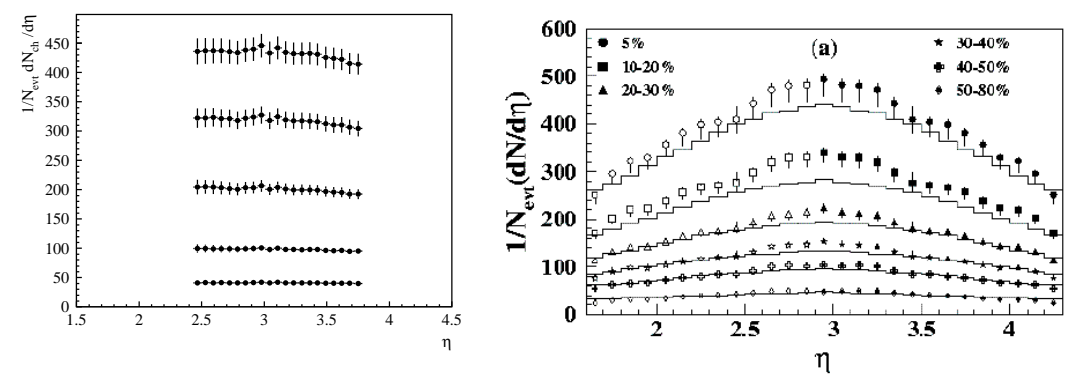

Figure 2. Pseudorapidity distributions of charged particles and photons in $\mathrm{Pb}$ induced reactions at $158 \cdot A \mathrm{GeV}$ on a $\mathrm{Pb}$ target. The charged particle distributions shown in the left panel are for $0-10 \%, 10-20 \%, 20-40 \%, 40-60 \%$ and $60-80 \%$ centrality bins. The centrality bins for photons are as labelled in the figures.

hadron rejection threshold are identified as photon-like $\left(N_{\gamma \text {-like }}\right)$. The photon counting efficiencies for central to peripheral cases vary from $68 \%$ to $73 \%$ [18, 19]. The purity of the photon sample in the two cases varies from $65 \%$ to $54 \%$. The centrality of the interaction is determined by the total transverse energy $\left(E_{\mathrm{T}}\right)$ measured in the mid rapidity calorimeter. The centralities are expressed as fractions of the measured total transverse energy. The most central corresponds to the top $5 \%$ of the minimum bias cross section. Extreme peripheral events in the $80-100 \%$ range were not analyzed.

Fig. 11 shows the minimum bias charged particle and photon-like distributions within the full acceptance of the detectors. The distributions corresponding to the centrality cuts of $1 \%, 2 \%$ and $5 \%$ of minimum bias cross section are superimposed on the figure.

The pseudorapidity distribution of charged particles and photons at different centralities are shown in the left and right panels of Fig. 2, respectively. The data have been corrected for geometry and efficiency factors. For photons, the filled symbols represent the measured data, and the open symbols are reflections of the filled symbols at $\eta_{c . m} .(=2.92)$. The solid histograms show the corresponding distributions obtained from VENUS event generator [20.

\section{Scaling of particle production}

The gross features of particle production in nucleon-nucleon collisions and reactions of light nuclei are well described in the framework of wounded nuclear model [21]. In this model the transverse energy and particle production in $\mathrm{p}+\mathrm{A}$ and $\mathrm{A}+\mathrm{A}$ reactions are calculated by assuming a constant contribution from each participating nucleon. While a scaling with the number of collisions arises naturally in a picture of a superposition of nucleon-nucleon collisions, with a possible modification by initial state effects, the scaling using number of participants is more naturally related to a system with strong final state rescattering, where the incoming particles loose their memory and every participant contributes a similar amount of energy to particle production. The scaling behavior of particle production may therefore carry important information on the reaction dynamics 22. 


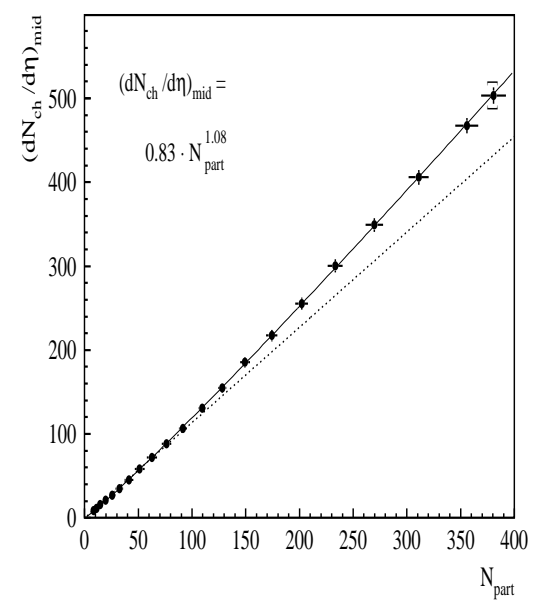

Figure 3. Scaling behavior of charged particles and photons. Pseudorapidity density of charged particles at midrapidity and integrated number of photons are plotted as functions of number of participating nucleons. The solid lines show power-law fit to the data, which yields the value of the exponent, $\alpha=1.07 \pm 0.05$ for charged particles and $1.12 \pm 0.03$ for photons, respectively.

The number of participants have been calculated using the VENUS model. Pseudorapidity density of $N_{\text {ch }}$ at midrapidity as a function of the number of participants for $\mathrm{Pb}+\mathrm{Pb}$ collisions are shown in the left panel of Fig. 3. The data points show stronger than linear increase (shown as dotted line). A scaling relation can be obtained by fitting the data points using $C \times N_{\text {part }}^{\alpha}$. The value of $\alpha$ is extracted to be $1.07 \pm 0.05$ [22]. Similarly on the right side we have shown the integrated number of photons as a function of number of participating nucleons. A similar power law fit yields the value of $\alpha$ to be $1.12 \pm 0.03$ [18]. The results for charged particles and photons are consistent with each other within the quoted errors. These results show that there is a clear participant scaling violation compared to a purely linear dependence. The scaling violation might have consequences on many other signals, for example, on the $J / \psi$ production.

\section{Multiplicity Fluctuations}

A lot of theoretical interest has been generated on the subject of event-by-event fluctuations, primarily motivated by the near perfect Gaussian distributions of several observables for a given centrality bin. If the distribution of a quantity $X$ is Gaussian, then one defines the amount of fluctuation by the following:

$$
\omega_{X}=\frac{\sigma_{X}^{2}}{<X>}
$$

where $\sigma_{X}$ is the variance of the distribution. That is, the fluctuation of the distribution is the variance squared normalized to the mean of the distribution under consideration.

In Fig. 1, the distributions of $N_{\mathrm{ch}}$ and $N_{\gamma \text {-like }}$ are shown for centrality bins of $0-1 \%$, $0-2 \%$ and $0-5 \%$. These curves are very good Gaussians with fits giving $\chi^{2} /$ ndf to be close to unity. It has been observed that making the centrality bin broader beyond $0-5 \%$, (from 0-6\% and beyond) the distributions deviate from Gaussians. Using the mean and variance of the distributions at different centrality bins we have calculated the amount 

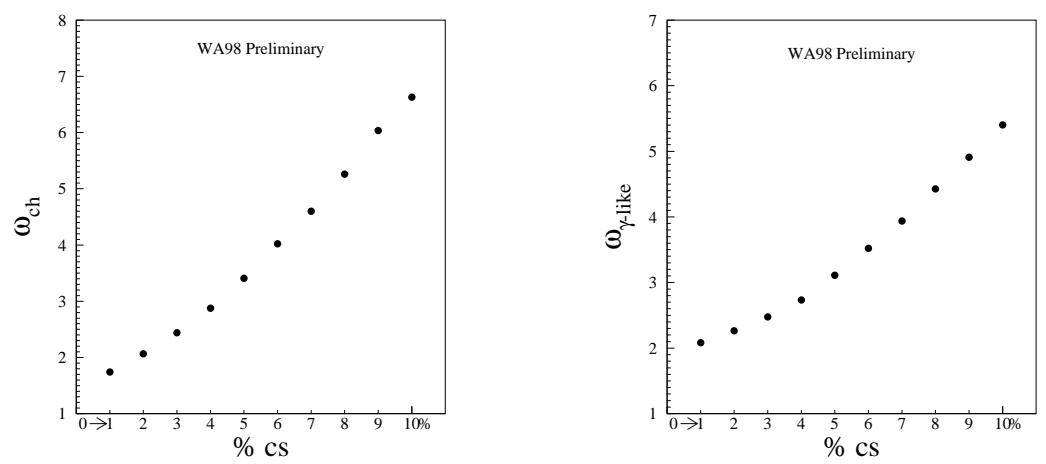

Figure 4. Multiplicity fluctuations of charged particles and photons for various centralities within the full coverage of the PMD and SPMD. The width of the centrality bins as percentage of minimum bias cross section increases along the X-axis.
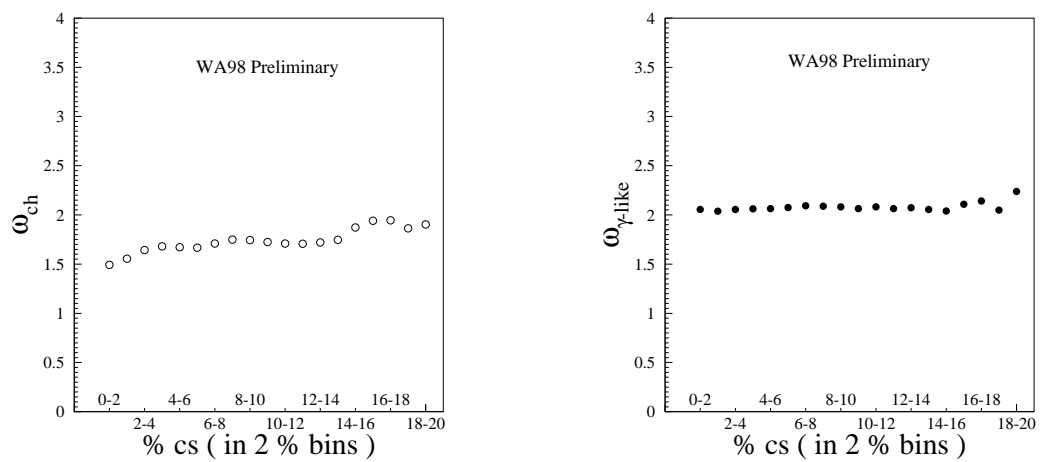

Figure 5. Multiplicity fluctuations of charged particles and photons for various centralities in a common coverage of the PMD and SPMD. The width of the centrality bins as percentage of minimum bias cross section remain same $(2 \%)$ along the $\mathrm{x}$-axis.

of fluctuations using eqn. (1). These results are plotted in Fig. 囵 for charged particles and photons. It is seen that the amount of fluctuation increases by increasing the width of the centrality bin. This is obvious from the fact that by increasing the width of the centrality selection bin, the inherent statistical fluctuations also increases.

Thus it is very important to control centrality properly in all fluctuation studies such that the multiplicity distributions are good Gaussians. Keeping this aspect in mind we have used a different set of centrality selection by taking $2 \%$ cross section bins, such as, 0-2\%, 2-4\%, 4-6\%, 6-8\%, etc. The resulting multiplicity distributions are good Gaussians in nature with $\chi^{2} /$ ndf between 1 and 1.5. The amount of fluctuations are calculated for these type of centrality bins and plotted in Fig. 5. The fluctuations for charged particles increase weakly by going from highest centrality towards peripheral whereas 

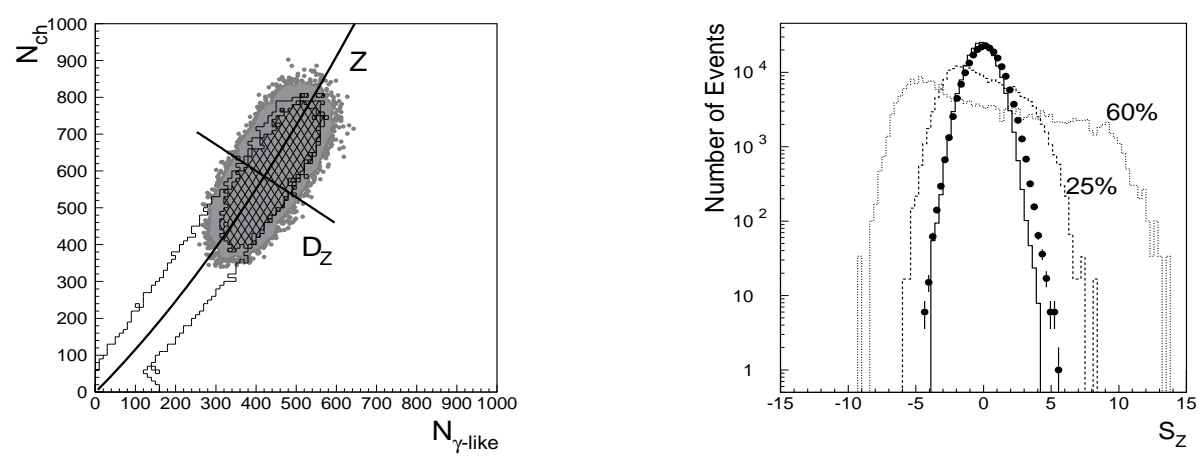

Figure 6. The left panel shown the correlation of $N_{\gamma-\text { like }}$ and $N_{c h}$ for central (top $10 \%$ ) events. The hatched region is for VENUS+GEANT simulation results. The right panel shows the $S_{Z}$ distribution for the experimental data is shown, overlaid with VENUS+GEANT results (solid histogram). The other curves are generated by incorporating $25 \%$ and $60 \%$ DCC in every event.

for photons the fluctuations are almost uniform. The fluctuations in photons are higher compared to those of the charged particles. This difference may arise because majority of photons are decay products of $\pi^{0}$. These results have to be put in perspective in terms of the contributions from known sources, such as, effect of finite multiplicity, fluctuations because of impact parameter of the reaction, effect of rescattering, BE correlations, and resonance decays.

\section{Charged-neutral fluctuations}

We now turn to event-by-event fluctuations in charged particle and photon multiplicities. We make a global event characterization in terms of the photon and charged particle distributions over the full available phase space of the detectors. The main motivation is to search for single large size DCC domains by looking for events which fall far beyond the correlation line of $N_{\gamma-\text { like }}-N_{\text {ch }}$ distributions.

The correlation plot of $N_{\gamma \text {-like }}$ and $N_{c h}$ for top $10 \%$ central events is shown in the left panel of Fig. 6. A strong correlation is seen between charged and neutral multiplicities. The fitted line of the distribution is represented by the $Z$ axis. The closest distance $\left(D_{Z}\right)$ of the data points to the correlation axis has been calculated numerically with the convention that $D_{Z}$ is positive for points below the Z-axis. We have chosen to work with the scaled variable $S_{Z} \equiv D_{Z} / \sigma_{D_{Z}}$ in order to compare relative fluctuations at different multiplicities. $S_{Z}$ distribution for the data is shown as filled circles in the right panel of Fig. 6. the solid histogram shows the results from the VENUS events passed through the GEANT simulation package of WA98 experiment. These events are termed as $(V+G)$ in rest of the manuscript. The DCC events are expected to show up as non-statistical tails in the distribution of $S_{Z}$. This is illustrated by the other curves where one introduces a known about of DCC fluctuation. Introduction of DCC makes the curves broader. Since 
we do not see any such event in our data sample, we are faced with the possibilities that single-domain DCCs are very rare, very small, or both. Based on a simple DCC model calculation we have put upper limits on the frequency of DCC production as a function of its size. More details may be obtained from [23]. We discuss search for small size DCC in the next section.

\section{Localized charged-neutral fluctuations}

After studying the charged-neutral fluctuations on the full available phase space, the next interest would be to search for fluctuations in localized $(\eta-\phi)$ phase space regions or domains [24]. One of the major interest would be the search for the formation of small size DCC as it is supposed to produce clusters of coherent pions in localized domains. The probability distribution of neutral pion fraction in a DCC domain follows the relation :

$$
P(f)=1 / 2 \sqrt{f} \quad \text { where } \quad f=N_{\pi^{0}} / N_{\pi} .
$$

Thus DCC formation in a given domain would be associated with large correlated eventby-event fluctuations in the multiplicities of charged particles and photons as majority of charged particles consist of charged pions and majority of the photons originate from $\pi^{0}$ decays.

The present analysis uses data from top $5 \%$ central events, which corresponds to a total of $85 \mathrm{~K}$ events. The pseudorapidity region common to both PMD and SPMD is selected. The acceptance in terms of transverse momentum $\left(p_{\mathrm{T}}\right)$ extends down to 30 $\mathrm{MeV} / \mathrm{c}$, although no explicit selection in $p_{\mathrm{T}}$ is applied. The experimental results are compared to simulated $\mathrm{V}+\mathrm{G}$ events and various types of mixed events.

\subsection{A simple DCC model}

The effect of non-statistical DCC-like charged-neutral fluctuations has been studied within the framework of a simple model in which the output of the VENUS event generator has been modified. In this, the charges of the pions within a localized $\eta$ - $\phi$ region from VENUS are interchanged pairwise $\left(\pi^{+} \pi^{-} \leftrightarrow \pi^{0} \pi^{0}\right)$, according to the DCC probability distribution as given in equation (2). For the present study, DCC events have been generated in the range of $3.0 \leq \eta \leq 4.0$ with varying intervals in $\Delta \phi$. An ensemble of events (henceforth referred to as $\mathrm{nDCC}$ events) were then generated by mixing different fractions of DCC-like events with those of normal events, as appropriate for different probabilities of occurrence of DCC. After allowing the $\pi^{0} \mathrm{~s}$ to decay, all the particles were then tracked through the full GEANT simulation program of WA98 experiment. These events are then analyzed using the same analysis methods as of the data.

Two different analysis methods have been used for the search of non-statistical fluctuations. The first one is the method of discrete wavelet transformation (DWT) and the second one is the $N_{\gamma-\text { like }-N_{\text {ch }}}$ correlation.

\subsection{Discrete wavelet transformation}

The DWT method [26] has been utilized very successfully in many fields including image processing, data compression, turbulence, human vision, radar, and earthquake prediction [25. The beauty of the DWT technique lies in its ability to analyze a spectrum at different resolutions with the ability to pick up any fluctuation present at the right scale. Simulation 
studies by Huang et. al. [13] and Nandi et. al. [16] have shown that the DWT analysis could be a powerful technique for the search of localized DCC.

While there are several families of wavelet bases distinguished by the number of coefficients and the level of iteration, we have used the $D-4$ basis [26]. The analysis has been performed with the sample function,

$$
f^{\prime}=\frac{N_{\gamma-\text { like }}}{N_{\gamma-\text { like }}+N_{\text {ch }}},
$$

calculated at the highest resolution scale, $j_{\max }=5$. The spectrum of $f^{\prime}$ is the input to the DWT analysis. The sample function is then analyzed at different scales $j$ by being re-binned into $2^{j}$ bins. The output of the DWT consists of a set of wavelet or father function coefficients (FFCs) at each scale, from $j=1, \ldots,\left(j_{\max }-1\right)$. The coefficients obtained at a given scale, $j$, are derived from the distribution of the sample function at one higher scale, $j+1$. The FFCs quantify the deviation of the bin-to-bin fluctuations in the sample function at that higher scale relative to the average behavior. The distribution of FFCs for normal events is Gaussian in nature. However, the presence of localized nonstatistical fluctuations makes the distribution broader, with a larger rms deviation of the FFC distribution 13, 15, 16. Comparing the rms deviations of the FFC distribution of data and mixed events one can get an idea about the localized fluctuations.

\section{3. $N_{\gamma}$ - like and $N_{\text {ch }}$ correlations}

The correlation between $N_{\gamma \text {-like }}$ and $N_{\text {ch }}$ has been studied in smaller $\phi$-segments by dividing the $\phi$-space into $2,4,8$ and 16 bins. The correlation plots of $N_{\gamma \text {-like }}$ and $N_{\text {ch }}$ have been constructed for each $\phi$ bin. This is shown in Fig. 7. A common correlation axis $(Z)$ has been obtained by fitting the above distributions with a second order polynomial. The closest distance $\left(D_{Z}\right)$ of the data points to the correlation axis has been calculated numerically with the convention that $D_{Z}$ is positive for points below the Z-axis. The distribution of $D_{Z}$ represents the relative fluctuations of $N_{\gamma \text {-like }}$ and $N_{\text {ch }}$ from the correlation axis at any given $\phi$ bin. In order to compare these fluctuations at different scales in the same level, we work with a scaled variable, $S_{Z}=D_{Z} / \operatorname{rms}\left(D_{Z}\right)$, where $\operatorname{rms}\left(D_{Z}\right)$ corresponds to VENUS events. The presence of events with localized fluctuations in $N_{\gamma-\text { like }}$ and $N_{\mathrm{ch}}$, at a given $\Delta \phi$ bin, is expected to result in a broader distribution of $S_{Z}$ compared to those for normal events at that particular bin.

\subsection{Mixed events}

In order to search for the presence of fluctuations in the experimental data, it is necessary to understand all detector related effects by generating different sets of mixed events, keeping specific physics goals in mind. Properly constructed mixed events should preserve all detector effects while removing correlations. Four sets of mixed events are generated to provide equivalent event samples as real events. In each type of mixed event the global (bin 1) $N_{\gamma \text {-like }}-N_{\text {ch }}$ correlation is maintained as in the real event.

The first type of mixed events (M1) are generated by mixing hits on both the PMD and SPMD hits separately, with no two hits taken from the same detector. Hits within a detector in the mixed events are not allowed to lie within the two track resolution of that detector. The second kind of mixed events (M2) are generated by mixing unaltered PMD hits of one event with the unaltered SPMD hits of a different event. Two more mixed 


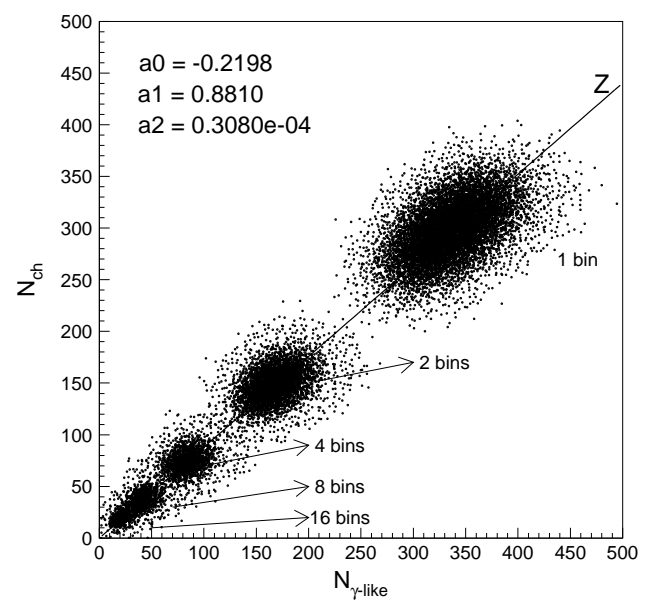

Figure 7. The scatter plot showing the correlation of $N_{\gamma-\text { like }}$ and $N_{\text {ch }}$ for $1,2,4,8$ and 16 bins in azimuthal angle, $\phi$, for a common coverage of the two detectors, PMD and SPMD. The data are for central events, corresponding to top $5 \%$ of the minimum bias cross section. The scatter plots at different bins are fitted to a curve (shown as Z axis) with a second order polynomial. The fit parameters are shown.

Table 1

Types of mixed events and how they are used for different physics.

\begin{tabular}{|cccc|}
\hline & PMD & SPMD & Type of fluctuation to probe: \\
\hline M1 & Mix hits & Mix hits & Correlated + Individual \\
M2 & Unaltered hits & Unaltered hits & Correlated \\
M3- $\gamma$ & Unaltered hits & Mix hits & $N_{\gamma}$ only \\
M3-ch & Mix hits & Unaltered hits & $N_{\text {ch } \text { only }}$ \\
\hline
\end{tabular}

event types are possible which are intermediate between the M1 and M2 mixed events: (i) the hits within the PMD are unaltered while the SPMD hits are mixed, this is called M3- $\gamma$, and (ii) the SPMD hits are unaltered while the PMD hits are mixed, this is called M3-ch. In table 1 we summarise the construction and usefulness of each of the mixed events.

The behavior of the mixed events may be understood from the sample of nDCC events. We have constructed all four different kinds of mixed events from the nDCC events and analyzed using both the methods. Here we give the results of the DWT analysis. The rms deviations of the FFCs for $\mathrm{nDCC}$ events and the different kinds of mixed events produced from the $\mathrm{nDCC}$ events are shown in Fig. 8. In the absence of DCC-like fluctuation the rms values of the various types of mixed events are very close to each other. But the $\mathrm{V}+\mathrm{G}$ rms values come out to be lower than those of the mixed events. This is due to the presence of additional correlations between $N_{\mathrm{ch}}$ and the charged particle contamination 


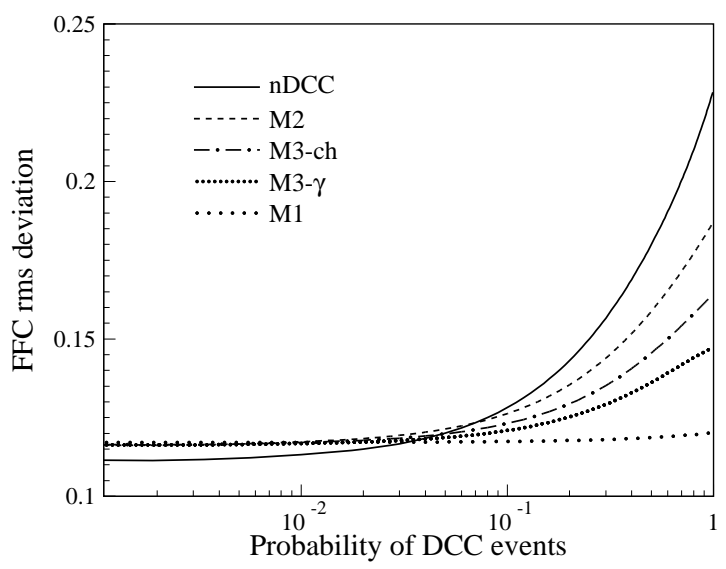

Figure 8. The rms deviations of the FFC distributions at $j=1$ for simulated nDCC events with extent $\Delta \phi_{D C C}=90^{\circ}$ and for various mixed events constructed from those events, as a function of the fraction of DCC-like events present in the nDCC sample.

in the $N_{\gamma-\text { like }}$ sample.

The rms deviations for the M1-type of mixed events are found to be almost independent of probability of DCC-like events. Thus it provides a baseline for studying non-statistical fluctuations. The rms deviations of the M2 type of mixed events increase similarly, but more weakly, than those of the nDCC events. The rms deviations for the M3 sets of events are found to lie between M2 and M1. Thus, the sequence of the mixed events relative to the simulated nDCC events (or data) gives a model independent indication of the presence and source of non-statistical fluctuations. The simple DCC model used here results in an anti-correlation between $N_{\gamma-\text { like }}$ and $N_{\text {ch }}$ due to the "isospin-flip" procedure used to implement the DCC effect. It also results in non-statistical fluctuations in both $N_{\gamma \text {-like }}$ and $N_{\text {ch }}$. Thus the M2 mixed events remove only the $N_{\gamma-\text { like }}-N_{\text {ch }}$ anti-correlation while the M1 mixed events are seen to remove all non-statistical fluctuations and correlations. The M3 mixed events give intermediate results because they contain only the $N_{\gamma \text {-like }}$ (M3- $\gamma$ ) or $N_{\text {ch }}$ (M3-ch) non-statistical fluctuations.

\subsection{Results}

The $S_{Z}$ distributions calculated at 4 and 8 bins in $\phi$ angle are shown in the top panel of Fig. 9 for data, M1 and $\mathrm{V}+\mathrm{G}$ events. The experimental data is broader than the simulation and M1 events, indicating the presence of additional fluctuations. The FFC distributions extracted from the measured $f^{\prime}(\phi)$ ratio are shown in the bottom panel of Fig. 9 for the experimental data, for M1-type mixed events (from data), and for $\mathrm{V}+\mathrm{G}$ events. The results are shown for scales $j=1$ and 2, which carry information about fluctuations at $90^{\circ}$ and $45^{\circ}$ in $\phi$, The FFC distributions of the experimental data are seen to be broader than both the mixed and $\mathrm{V}+\mathrm{G}$ events. The result again suggests the presence of non-statistical fluctuations.

The rms deviations of the $S_{Z}$ and FFC distributions as a function of the number of bins in azimuth is shown for experimental data, mixed events, and $V+G$ in Fig. 10. The 

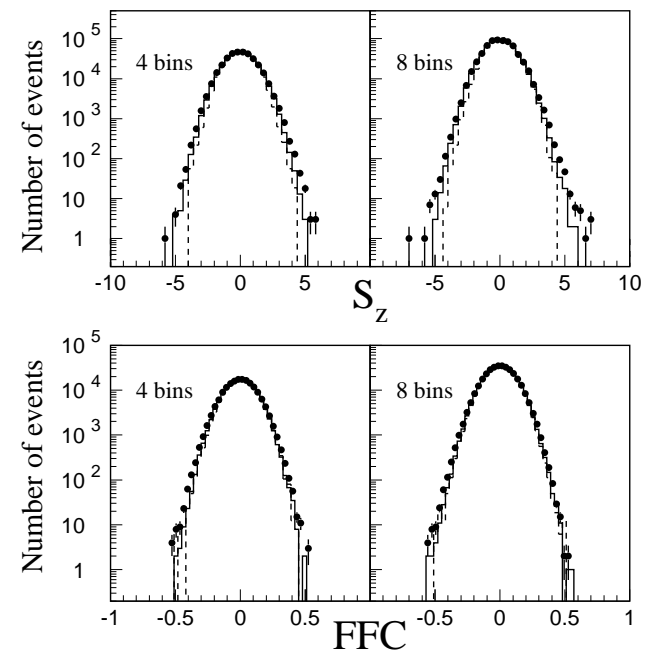

Figure 9. The $S_{Z}$ and FFC distributions for 4 and 8 divisions in $\phi$. The experimental data, M1 and V+G events are shown by solid circles, solid histograms and dashed histograms, respectively. The statistics for data and mixed events are the same, whereas the distribution for the $\mathrm{V}+\mathrm{G}$ events is normalized to the number of data events.

statistical errors on the values are small and lie within the size of the symbols. The error bars include both statistical and systematic errors. The systematic errors include effects such as uncertainty in the detection efficiencies, gain fluctuations, backgrounds, binning variations and fitting procedures. The total systematic error was obtained as the sum in quadrature of the individual error contributions.

Since the mixed events are constructed to maintain the $N_{\gamma-\text { like }}-N_{\text {ch }}$ correlations for the full azimuth (bin 1), the rms deviations of data and mixed events for this bin are identical. The difference of the rms deviations between data and $\mathrm{V}+\mathrm{G}$ for this bin is the same as shown in the right hand panel of Fig. 6 23]. The effect of correlations due

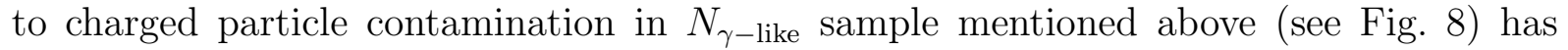
been removed by rescaling the mixed event results according to the percentage difference between the rms deviations of the $S_{Z}$ and FFC distributions of $\mathrm{V}+\mathrm{G}$ events and those of the corresponding $\mathrm{V}+\mathrm{G}$ mixed events.

Comparison of the rms deviations of the experimental data and mixed events have been made for all the three different cases.

- (1) Comparison with M1 : From Fig. 10 one notices that the data points are higher compared to those of the M1 events for several bins in $\phi$. The differences between the data points and M1 mixed events have been quantified by using the quantity, $\sigma=\sqrt{\left(\sigma_{\text {lower }}^{\text {data }}\right)^{2}+\left(\sigma_{\text {upper }}^{M 1}\right)^{2}}$. For 2,4 , and 8bins the values of the $S_{Z} \mathrm{rms}$ deviations of the data are $2.5 \sigma, 3.0 \sigma, 2.4 \sigma$ larger compared to those of the M1 events, respectively. Similarly the rms deviations of the FFC distributions at 4 and 8 bins for data are $3.7 \sigma$ and $2.8 \sigma$ larger than those of the M1 events. At 16 and 32 bins the results for mixed events and data agree within the quoted errors. 

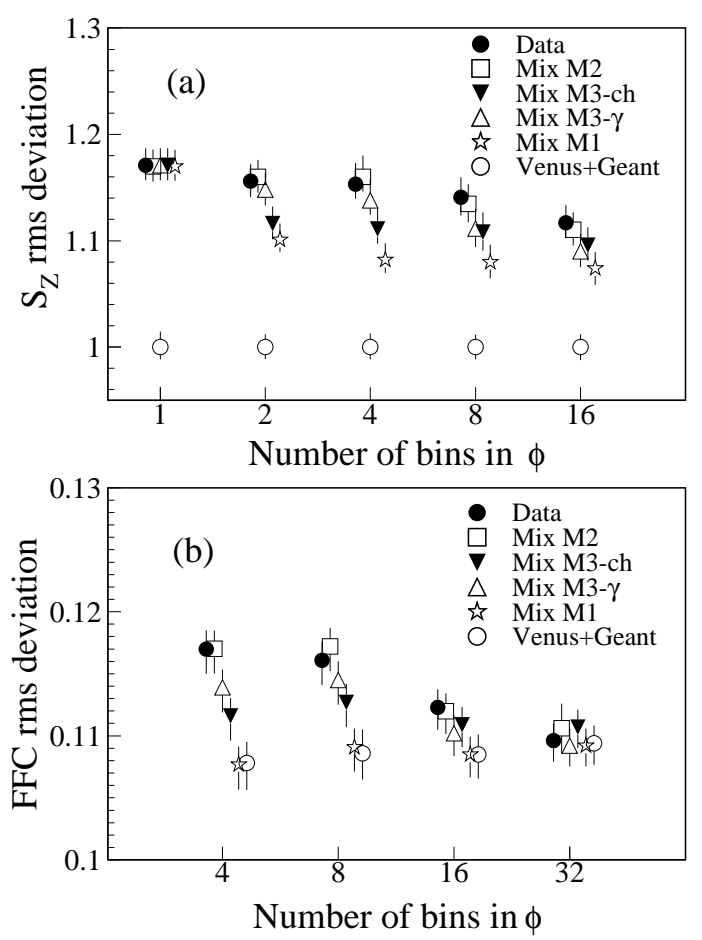

Figure 10. The root mean square (rms) deviations of the $S_{Z}$ and FFC distributions for various divisions in the azimuthal angle.

This indicates the presence of localized non-statistical fluctuations. This result is completely model independent as the comparison has been made to mixed events generated from data. The observed non-statistical fluctuations may arise because of (a) event-by-event correlated $N_{\gamma \text {-like }}-N_{\text {ch }}$ fluctuations, (b) individual fluctuations in $N_{\gamma \text {-like }}$ and (c) individual fluctuations $N_{\mathrm{ch}}$. The source of the fluctuation can be obtained by comparing the data with the results from M2, M3- $\gamma$ and M3-ch.

- (2) Comparison with M2 : From Fig. 10 it is evident that the rms deviations of the M2 events agree with those of the experimental data within errors for all bins in $S_{Z}$ and FFC distributions. This implies the absence of event-by-event correlated fluctuations in $N_{\gamma-\text { like }}$ versus $N_{\text {ch }}$.

If the amount of DCC-like fluctuations in the experimental data were large, then the rms deviations for data would have been larger than those of M2 events. Since this is not the case, we have extracted upper limits on the probability of DCC-like fluctuations at the $90 \%$ confidence level by comparing the results from data with those obtained from the nDCC events. Using the DCC-model used in this analysis we have extracted upper limits on the probability of DCC events to be $10^{-2}$ for $\Delta \phi$ between $45-90^{\circ}$ and $3 \times 10^{-3}$ for $\Delta \phi$ between $90-135^{\circ}$.

- (3) Comparison with M3- $\gamma$ and M3-ch : The M3 type mixed events shown in Fig. 10 are found to be similar to each other within the quoted errors and lie between M1 and M2. This indicates the presence of localized independent fluctuations in $N_{\gamma-\text { like }}$ and $N_{\mathrm{ch}}$. 


\section{Summary}

Multiplicity distributions and fluctuations are important for understanding the evolution of nuclear systems at high energy collisions. These have been studied from the multiplicity measurements of charged particles and photons in $158 \cdot A \mathrm{GeV} \mathrm{Pb}+\mathrm{Pb}$ reactions. Production of charged particles and photons over the full range of centrality could be described in terms of participant scaling as $N_{\text {part }}^{1.07 \pm 0.05}$ and $N_{\text {part }}^{1.12 \pm 0.03}$, respectively. This indicates violation of the naive wounded nucleon model. Multiplicity fluctuations have been studied by varying the centrality of the reaction. It has been shown that the centrality of the reaction has to be properly chosen so that the multiplicity distributions are of good Gaussians. We have shown that this criterion is satisfied by making narrower bins in $E_{\mathrm{T}}$ corresponding to $2 \%$ of minimum bias cross sections. The fluctuations for photons are constant $(\omega=2.0 \pm 0.06)$ over the centrality range considered and are higher than those for charged particles $(\omega=1.8 \pm 0.11)$.

Event-by-event charged to neutral fluctuations have been studied in full acceptance of the detectors and in localized $(\eta-\phi)$ phase space. Using global event characterization, no event with large charged-neutral fluctuations have been observed. A mixed event analysis is not possible for this global search. The search for localized fluctuations have been carried out by comparing data with mixed events generated from the data. Full understanding of the nature of the mixed events have been achieved by using a simple DCC model. Two different analysis methods, (1) $N_{\gamma \text {-like }}$ and $N_{\mathrm{ch}}$ correlation method, and (2) a more sophisticated DWT method, have been employed. Both analysis methods provide model-independent evidence for non-statistical fluctuations at the $3 \sigma$ level for $\phi$ intervals greater than $45^{\circ}$. This is seen to be due to non-statistical fluctuations in both both $N_{\gamma}$ and $N_{\mathrm{ch}}$. No significant event-by-event correlated fluctuations are observed, contrary to expectations for a DCC effect. The origin of the observed individual fluctuations are not known at present. The interpretation of the results remains an open question.

With much higher particle multiplicities achieved at higher energies of RHIC and LHC, analysis methods using event-by-event fluctuations would certainly be very essential to probe the signals of QGP and DCC. Better understanding of data at SPS energies would definitely help in making firmer conclusions of any signal from future experiments.

\section{REFERENCES}

1. Proceedings of Quark Matter '99, Nucl. Phys A 661 (1999).

2. Proceedings of Quark Matter 2001, Stoney Brook, USA, to be published in Nucl. Phys. A.

3. E.V. Shuryak and O. Zhirov, Phys. Lett. B89 (1980) 253.

4. L. van Hove, Phys. Lett. B118 (1982) 138.

5. M. Stephanov, et al., Phys. Rev. Lett. 81, 4816 (1998).

6. Gordon Baym and Henning Heiselberg, Phys. Lett. B469, 7 (1999).

7. M.Asakawa, U.Heinz, and B.Müller, Phys. Rev. Lett. 85, 2072 (2000).

8. S. Jeon and V. Koch, Phys. Rev. Lett. 85, 2076 (2000).

9. A.A. Anselm, M.G. Ryskin, Phys. Lett. B 266, 482 (1991).

10. J.D. Bjorken, Int. J. Mod. Phys. A 7, 4189 (1992). J.D. Bjorken, K.L. Kowalski, C.C. Taylor, "Baked Alaska", SLAC-PUB-6109, Apr. 1993. 
11. J. -P. Blaizot and A. Krzywicki, Phys. Rev. D 46, 246 (1992).

12. K. Rajagopal and F. Wilczek, Nucl. Phys. B 399, 395 (1993); Nucl. Phys. B 404, 577 (1993).

13. Z. Huang, et al., Phys. Rev. D 54, 750 (1996).

14. T.K. Nayak, WA98 Collaboration, Nucl. Phys. A638, 249c (1998).

15. B.K. Nandi, G.C. Mishra, B. Mohanty, D.P. Mahapatra and T.K. Nayak, Phys. Lett. B449 (1999) 109.

16. B.K. Nandi, T.K. Nayak, B. Mohanty, D.P. Mahapatra and Y.P. Viyogi, Phys. Lett. B461 (1999) 142.

17. J. Randrup, Phys. Rev. C 62, 064905 (2000).

18. WA98 Collaboration, M.M. Aggarwal et al., Phys. Lett. B 458, 422 (1999).

19. M.M. Aggarwal et al., Nucl. Instr. and Meth. A 424, 395 (1999).

20. K. Werner, Phys. Rep. 232, 87 (1993).

21. A. Bialas, A. Bleszynski and W. Czyz, Nucl. Phys. B 111, 2000 (1976).

22. WA98 Collaboration, M.M. Aggarwal et al., Eur. Phys. J C18, 651 (2001).

23. WA98 Collaboration, M.M. Aggarwal et al., Phys. Lett. B 420, 169 (1998).

24. WA98 Collaboration, M.M. Aggarwal et al., e-print archive: nucl-ex/0012004.

25. A Graps, IEEE Computational Sciences and Engineering, 2, 50 (1995).

26. Numerical Recipes, Cambridge Univ. Press , 1998. 